\title{
Calcium does not impair iron status in practice
}

$\mathrm{S}$ hort-term studies with isotope-labelled iron have shown that calcium may inhibit iron absorption from meals, and molecular mechanisms for such an inhibition have been identified recently. The inhibition seems to be maximal with 150-200 mg calcium in a meal. However, mechanisms regulating the iron status through absorption have also been increasingly elucidated and may counteract this inhibition, especially if rather large amounts of calcium are consumed in the long term.

Long-term trials with calcium supplementation, carried out primarily to study effects on bone mineral density, have failed to confirm any impairment of calcium absorption on iron status. Therefore, interference with iron absorption is not a good reason to avoid drinking milk with main meals. This conclusion was drawn at a scientific workshop arranged recently by the Swedish Dairy Association (meeting report available in Swedish).

However, there is no reason to encourage drinking milk with every meal. Milk is a liquid nutritious food and the total amount in the diet should be considered in relation to the overall nutrient content and balance. Milk consumption has decreased in Sweden, but cheese has increased, with the average total intake of dairy products corresponding to about $9 \mathrm{dl}$ milk per day. This exceeds the recommended $5 \mathrm{dl}$ per day. Rather than encouraging an overall increase in milk consumption, there should be a focus on risk groups avoiding milk, especially teenagers and young women, who may have a suboptimal calcium intake.

\section{Is calcium important in body weight regulation?}

I ncreased calcium intake with dairy products or supplements has been associated with weight loss in epidemiological studies, and animal experiments support a role of calcium in body weight regulation. An effect on energy balance by decreased fat absorption due to calcium soap formation in the intestine has been suggested, as well as an influence on energy metabolism at the cellular level.

A systematic review and meta-analysis of randomized controlled trials with calcium supplements or increased provision of dairy products (at least $300 \mathrm{mg}$ extra calcium per day) was published recently (1). Studies for 12 weeks or more in nonpregnant, non-lactating individuals aged at least 18 years were included. The meta-analysis was based on 13 studies, nine with calcium supplementation and four with increased intake of dairy products. No association was found between the increased consumption of calcium from supplements or dairy products and weight loss after adjustments for differences in baseline weights between the study and control groups. The overall conclusion was that calcium supplementation has no statistically significant association with a reduction in body weight.

Although several studies, on both humans and experimental animals, indicate that dietary calcium may be involved in body weight regulation, it is too early to promote calcium supplements or dairy products as slimming agents. More properly powered intervention studies specifically aimed at testing this hypothesis are needed before any conclusions can be drawn in practice.

\section{Reference}

1. Trowman R, Dumville JC, Hahn S, Torgerson DJ. A systematic review of the effects of calcium supplementation on body weight. Br J Nutr 2006; 95: 1033-8.

Nils-Georg Asp

SNF Swedish Nutrition Foundation

Lund

Sweden

E-mail: asp@snf.ideon.se 